\title{
The modified dynamics as a vacuum effect
}

\author{
Mordehai Milgrom \\ Department of condensed-matter physics, Weizmann Institute, Rehovot Israel
}

\begin{abstract}
To explain the appearance in MOND of a cosmological acceleration constant, $a_{0}$, I suggest that MOND inertia-as embodied in the actions of free particles and fields-is due to effects of the vacuum. The same vacuum effects enter both MOND (through $a_{0}$ ) and cosmology (e.g. through a cosmological constant $\Lambda$ ). For example, a constant-acceleration $(a)$ observer in de Sitter universe sees Unruh radiation of temperature $T \propto\left[a^{2}+a_{0}^{2}\right]^{1 / 2}$, with $a_{0} \equiv(\Lambda / 3)^{1 / 2}$, and I note that $T(a)-T(0)$ depends on $a$ in the same way that MOND inertia does.
\end{abstract}

\section{INTRODUCTION}

The modified dynamics (MOND) has been propounded [1] as an alternative to the dark-matter doctrine, attributing the mass discrepancy evinced by galaxies and galaxy systems to a breakdown of standard dynamics. The pristine version of MOND states that dynamics is to be modified in the limit of small accelerations, such as are found in galactic systems. (This is analogous to viewing quantum mechanics as a modification of classical mechanics in the limit of small angular momenta.) More precisely, MOND asserts that non-relativistic dynamics involves the constant $a_{0}$, with the dimensions of acceleration, so that in the formal limit $a_{0} \rightarrow 0$-i.e., when all quantities with the dimensions of acceleration are much larger than $a_{0}$-standard dynamics obtains. In the opposite (MOND) limit of large $a_{0}$ dynamics is marked by reduced inertia. One may roughly say that in this limit inertia at acceleration $a$ is $m a^{2} / a_{0}$, instead of the standard $m a$. (In the quantum analogue, mechanics involves some constant, $\hbar$, with the dimensions of angular momentum, such that classical mechanics prevails in the formal limit of small $\hbar$.)

The idea has been successfully tested against astronomical data (see e.g. [2] [3] [4]) but still wants firm theoretical underpinnings. The value of $a_{0}$ that emerges from such analyses $\left(\approx 10^{-8} \mathrm{~cm} \mathrm{~s} s^{-2}\right)$ is of the order of $c H_{0}\left(H_{0}\right.$ is the Hubble constant) which is an acceleration of cosmological significance [1]. Even if $a_{0}$ is a fingerprint of cosmology on local dynamics, it is not necessarily a proxy for $a_{e x} \equiv c H_{0}$. There are other cosmological acceleration scales that one can define [5] [6]; e.g., $a_{c} \equiv c^{2} / R_{c}$, where $R_{c}$ is the curvature (spatial or space-time), or $a_{\Lambda} \equiv c \Lambda^{1 / 2}$, where $\Lambda$ is the cosmological constant. Today we have only an upper limit on $a_{c}$ that is of the order of $a_{e x}$, and recent evidence (see e.g. [7, 8]) points to a nonzero cosmological constant of order $H_{0}^{2}$, for which $a_{0} \sim a_{\Lambda}$. So $a_{0}$ might be a proxy for any of these cosmological parameters. The exact identification has important conceptual and phenomenological consequences, e.g. because the different acceleration scales depend differently on cosmic time. If we make such a connection with cosmology, we see that in a "trivial" universe-one that is flat, static, and has a vanishing cosmological constant $-a_{0}$ would vanish, and standard dynamics be restored.

In relativity theory, inertia and gravity are intertwined. This might turn out to be true also in the ultimate theory for MOND. However, in the undeveloped state in which the theory is now it is customary to think of MOND as either a modification of gravity (e.g. [9]) or as of one of inertia (e.g. [6]). The difference between the two options is not only one of principle. One can expect major differences in predictions regarding the dynamics of astrophysical systems [6].

Here, I shall concentrate on the modified-inertia interpretation of MOND. The line I would like to follow is that inertia is a derived property of matter that results from the interaction of matter with some agent-the vacuum, in the present case. Cosmology either affects or is affected by the vacuum. Inertia is thus influenced by, or shares common influences with, cosmology; and, it is through this that $a_{0}$ enters local dynamics and cosmology. In other words, either cosmology has a causative effect on inertia because it affects the state of the vacuum, which, in turn, affects inertia, or, cosmology and inertia are both affected by the vacuum dynamics, which then enters cosmology say as a cosmological constant, $\Lambda$, and MOND through $a_{0} \approx c \Lambda^{1 / 2}$.

The same connection could come about in MOND as modified gravity; at present I deem modified inertia a more promising avenue.

Inertia was introduced into physics as an attribute that endows bodies with energy and momentum according to their motion, and these can be changed only by subjecting the body to a force. Attempts to generate inertia-in the spirit of Mach's principle-have traditionally concentrated on inertia of bodies (see e.g. 10]). More generally, however, inertia is the attribution of energy and momentum to all dynamical degrees of freedom, whether we describe them as bodies (particles) or fields. This attribution is conventionally encapsuled in the action of the free particles and field, from which the energy-momentum tensor is gotten. To derive inertia it might behoove us then to derive the free actions of matter fields. Alternatively, we might start from the equation of motion of fields, and try to derive its 
free part as some sort of force exerted by the vacuum. Apart from occasional comments I shall limit myself in what follows to the discussion of the archetypal case of inertia of bodies.

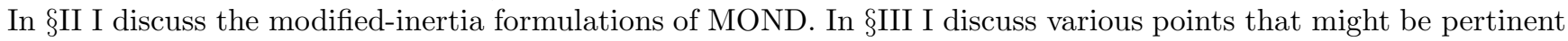
to inertia as a vacuum effect, and in $§ I V ~ I$ add some comments that are specific to MOND inertia.

\section{MOND AS MODIFIED INERTIA}

Assuming that MOND is underpinned by an action-which is not at all obvious-a possible intermediate stage in formulating MOND as modified inertia is to look for an effective kinetic action that governs the motion of a body. If $\mathbf{r}(t)$ is the trajectory of the body, we want the kinetic action to be of the form

$$
A_{m} S\left[\mathbf{r}(t), a_{i}\right]
$$

such that $A_{m}$ depends only on the body, and $S$ depends only on the trajectory $\mathbf{r}(t)$ and on the parameters $a_{i}$ measuring the departure from standard physics, and presumably connected with cosmology. The coefficient $A_{m}$ can then be identified as the rest mass of the body (gravitational and inertial) as the body's contribution to the energymomentum tensor will be $A_{m} t^{\mu \nu}$ with $t^{\mu \nu}$ depending only on the trajectory. In the limit of "trivial" cosmology, we would want $S$ to go to the standard expression $\int d \tau$. The interaction action remains intact by MOND according to this picture.

In general, all aspects of cosmology might enter $S$. However, almost all the astrophysical systems studied in light of MOND to date (galaxies of all sorts, galaxy groups, and clusters) are characterized by very nonrelativistic motions, by sizes much smaller than the the cosmological scale, and by dynamical times smaller than the Hubble time. For such systems the approximate statement of MOND would be that cosmology is connected to local dynamics only through the one parameter $a_{0}$, which is of the order of the accelerations found in these systems. The approximate effective action is thus written as

$$
A_{m} S\left[\mathbf{r}(t), a_{0}\right] .
$$

This, as I said, need not be the action for bodies still partaking in the Hubble flow, or that are relativistic; there, cosmology might enter in a more elaborate manner. In the limit $a_{0} \rightarrow 0, S$ should go to the standard expression

$\lim _{T \rightarrow \infty} T^{-1} \int_{-T}^{T} v^{2} / 2 d t$. In the opposite limit, MOND phenomenology dictates that $S$ scale as $a_{0}^{-1}$. In particular, an important tenet of MOND is that inertia vanishes as $a_{0} \rightarrow \infty$ (see below for an explanation of this in our Machian scheme).

Actions of the form (2) have been studied in detail in [6]. It was shown, for instance, that Galilei invariance, combined with the two limits in $a_{0}$, requires that $S$ be a nonlocal (in time) functional of the trajectory. This should not be viewed as an imposition. On the contrary, nonlocality appears naturally in effective action as ours must be. Also, nonlocality might be a blessing as nonlocal theories need not suffer from the maladies that are endemic to higher-order theories ( [6] and references therein). Nonlocality also opens the way to solving, in modified-inertia versions, the center-of-mass motion of composite bodies (the problem is solved in the modified-gravity version of ref. [9]): MOND inertia is nonlinear; how then do the equations of motion for constituents combine to give the correct equation of motion for the center-of-mass motion? This question comes up at the microscopic level of inertia-fromvacuum models, and also at the macroscopic level [1]. For instance, atoms inside stars have high accelerations, yet for MOND to work we want these stars, which move with low accelerations in galaxies, to follow MOND inertial behavior. The problem can probably not be solved in local theories (i.e. when the inertia force depends only on a finite number of time derivatives of the trajectory). In nonlocal theories this is not the case. Consider, for example, a heuristic model in which the equation of motion for a particle moving in a potential $\varphi$ is of the form

$$
\omega^{2} \mathbf{R}(\omega) \mu\left[|\mathbf{A}(\omega)| / a_{0}\right]=\mathbf{D}(\omega),
$$

where we work in Fourier space at frequency $\omega, \mathbf{R}(\omega)$ is the transform of $\mathbf{r}(t), \mathbf{D}(\omega)$ is the transform of $-\vec{\nabla} \varphi[\mathbf{r}(t)]$, and

$$
\mathbf{A}(\omega) \equiv \int_{0}^{\omega} \hat{\omega}^{2} \mathbf{R}(\hat{\omega}) d \hat{\omega}
$$

is a measure of the accumulated acceleration up to frequency $\omega$. (This equation of motion is not derivable from an action.) Conventional dynamics corresponds to the choice $\mu \equiv 1$. It is straightforward to see that, indeed, in this 
theory the center of mass (CM) motion of a body is hardly affected by internal motions inside it. If the accelerations characterizing internal motion are higher than those for the CM motion their frequencies must also be higher (because $a \sim \omega^{2} r$, and $r$ is smaller); these internal components do not then enter $\mathbf{A}$ for the CM motion. The CM motion does enter internal dynamics strongly in what is known in MOND as the external-field effect [1]. One can demonstrate all this by solving exactly equations (3) (4) for a potential that is harmonic in the $x-y$ plane, and linear in the $z$ direction: $\varphi=\Omega^{2}\left(x^{2}+y^{2}\right) / 2+g z$. One finds that the $z$ motion is of constant acceleration, which corresponds to frequencies near $\omega=0$, and is totally oblivious to the $x-y$ motion. The $x-y$ motion is still harmonic but with a frequency that depends on the amplitude and on the $z$ acceleration (and goes to $\Omega$ in the high-acceleration limit) exemplifying the external-field effect.

There are different MOND action of the form (2) that one could write, but no one, in particular, that deserves to be pinpointed as the "appropriate" action. They were all shown [6] to lead to an exact relation that governs circular motion in axisymmetric potentials (applicable to rotation curves):

$$
a \mu\left(a / a_{0}\right)=g_{N},
$$

where $a=v^{2} / r$ ( $v$ and $r$ are the orbital velocity and radius) $g_{N}$ is the Newtonian acceleration, and $\mu(x)$ is the MOND interpolating function, which here follows from the exact form of the action as restricted to circular orbits. It is this relation that has always been used in analyses of galaxy rotation curves (e.g. in [2] [3] [4]).

How might one derive such an effective action from more fundamental concepts, and, in particular, how does cosmology come to be connected with local dynamics in the MOND limit through $a_{0}$ ? It was suggested in [6] that this could come about if inertia is due-in the spirit of Mach's principle-to the effect of some agent, such as the vacuum, on non-inertial bodies. Since the vacuum is itself modified by the non-trivial state of the Universe, its inertia effect might also be imprinted with this information. Alternatively, it might be that it is not cosmology that affects local dynamics, but that the same vacuum attribute enters both cosmology, as a cosmological constant for instance, and MOND as $a_{0} \approx c \Lambda^{1 / 2}$.

In the next two sections I consider this surmise in more detail. I can offer no specific mechanism that begets MOND inertia from vacuum effects, but there is a number of pertinent ideas that might be of help in looking for such an underlying theory.

\section{INERTIA AS A VACUUM EFFECT}

As stated above, one way to generate inertia from effects of the vacuum is to derive the contribution of free fields to the action. In conventional dynamics these are $m \int d \tau$ for a free particle, $\int F_{\mu \nu} F^{\mu \nu}$ for the electromagnetic field, $\int\left(\partial_{\nu} f \partial^{\nu} \varphi\right)^{2}+m \varphi^{2}$ for a massive-scalar field, etc. MOND would contend that these are the approximate effective actions only in the limit of trivial cosmology, but, in general, effects of cosmology enter the action. In particular, in the nonrelativistic limit cosmology enters through $a_{0}$.

If the free action is to be derived from effects of the vacuum fields, then we must start with vacuum fields that themselves have no free action; we need to start with inter-field interactions only, and produce from these all the free actions.

It is well known that interactions can induce, or modify, inertial actions. For example, the effective mass of "free" electrons and holes in a semiconductor are greatly modified. More pertinent to our concern with the vacuum, mass renormalization in field theory is, of course, a vacuum effect. The Higgs mechanism induces an effective mass term from the interaction with the putative Higgs field. It is also known that the interaction of the electromagnetic field with charged vacuum fields induces a contribution to the free action of the electromagnetic field-the Heisenberg-Euler effective action (see e.g. [11], 12], and 13] p. 195). It is not clear whether, and exactly how, the mechanisms listed above fit the bill as concerns MOND. However, those that are known to affect inertia must be reckoned with in any complete analysis.

Sakharov [14] (see also [12] for a review) proposed a scheme to derive the "free" action of gravity from effects of the vacuum: Curvature of space-time modifies the dynamical behavior of vacuum fields, hence producing an associated energy or action for the metric field. To lowest order (in the Planck length over the curvature radius) this gives the Einstein-Hilbert action $\int g^{1 / 2} R$. Sakharov's arguments make use of the fact that the vacuum fields have inertia (since they are assumed to carry the usual energy-momentum). So, derived inertia comes prior to induced gravity a-la Sakharov. The two concepts are not, otherwise, related by the equivalence principle-as incorrectly stated in [15]. The Einstein-Hilbert action is not determined by the weak equivalence principle; the latter enters through the matter action alone (in dictating, e.g. that free particles move on geodesics, etc.). 
One can also bypass the action, start from the equation of motion, and try to derive the free part as a force exerted by the vacuum on non-inertial bodies. For instance, the Casimir effect may modify the inertia of macroscopic, as well as microscopic, bodies. In the least, the Casimir energy of a body should contribute to its inertial mass. Indeed, it was shown in 16] that two parallel mirrors accelerated together on an hyperbolic (linear, constant-acceleration) trajectory experience a force proportional to the acceleration, with a coefficient (induced mass) that corresponds to the Casimir energy of the double mirror. A Casimir energy stems from the energy-momentum of the vacuum fields, and so presupposes inertia of the vacuum fields. In a different attempt, it has been suggested in [15] that a body in acceleration is subject to a flux of radiation arising from the vacuum, and that if this flux is partially absorbed by the body, the momentum transferred might supply the inertia force on the body. Even if correct, this picture also tacitly presupposes that the vacuum fields themselves have inertia, because it builds on their carrying momentum, which they lose at impact (for instance, Maxwell dynamics is assumed for the vacuum electromagnetic field, which already implies inertia). Such mechanisms, which presuppose inertia, cannot be the primary origin of inertia. Yet another attempt [17 views each body as composed of charged oscillators that can oscillate only in the plane perpendicular to the acceleration-and here the alignment of the inertia force with the acceleration is put in by hand. The inertia force is then said to be a Lorentz-like force produced by the zero-point fluctuations on the constituent oscillators.

A precondition for a dynamical, inertia-producing effect of the vacuum is that an observer be able to perceive its non-inertiality through vacuum effects. It is indeed well known that for such an observer the vacuum transforms itself into a palpable radiation field. For an observer on a collinear trajectory of constant-acceleration, $a$, (hyperbolic motion) this is the Unruh radiation: a thermal bath of temperature $T=a \hbar / 2 \pi k c$ [18] [19]. (From here on I shall work in units where $\hbar=1, c=1, k=1$, so $T=a / 2 \pi$.) For a more general motion, the incarnation of the vacuum is non-thermal, and hardly anything seems to be known about the Unruh-like radiation. Some simple instances of stationary motion have been studied, e.g., in [20] [21]. In particular, circular, highly relativistic motions have been discussed in [21]- 25]. In this last case, as for hyperbolic motions, $a=\gamma^{2} v^{2} / r \approx \gamma^{2} / r$ determine the spectrum of the avatar of the vacuum ( $\gamma$ is the Lorentz factor). This is quasi-thermal with effective temperature $T=\eta a / 2 \pi$, where $\eta$ is of order unity and depends somewhat on the frequency. Circular motion in general is characterized by two parameters, which can be taken as $a$ and $r$. This leads to a rather more complicated Unruh effect, which has not been described yet. For stationary trajectories all points are equivalent, and so the Unruh-like radiation can be described in terms of "local" properties of the trajectory. Clearly, for a general motion the effect becomes a nonlocal functional of the whole trajectory.

Even at this basic level of the observer's need to read its motion in the vacuum it is not clear that he can read in it all that it needs. For instance can an observer in hyperbolic motion tell its direction, which would be necessary to define an inertia force? The Unruh flux itself is said to be isotropic [20], although there may be other attributes that the observer can pick up (e.g. fluctuations). Perhaps we need to take finite-size effects into account. For example, by comparing the Unruh radiation that its different parts see, the body could divine the direction of its acceleration.

It is thus doubtful whether the Unruh-like radiation itself is implicated in directly generating inertia. There is also the problem of response time: In standard dynamics inertia is simply proportional to the acceleration, but this may change on time scales that are shorter than the typical period of the Unruh radiation. It is claimed in [15] that their mechanism does give instantaneous adjustment because their putative flux of vacuum quanta appears to depend only on the instantaneous acceleration. This, however, cannot be correct if the acceleration changes on time scales that are not much longer than the typical period of the fields. For nonrelativistic, circular motion with velocity $v$ and orbital radius $r$ the typical wavelength of the Unruh radiation that corresponds to the acceleration is $\lambda=a^{-1}=r / v^{2} \gg r$, and the typical frequency of the radiation, $\omega=v \Omega \ll \Omega(\Omega$ is the angular frequency). So it is hard to see how the Unruh radiation itself can produce inertia that responds instantaneously to the the value of the acceleration, which we know must be the case at least in the non-MONDian regime. This could happen if in the non-MOND regime vacuum effects are dominated by the short-wavelength limit. If this is dictated, say, by a cutoff near the Planck scale, which is much smaller than any scale of relevance that we have probed so far, then instantaneous (local) inertia will follow. This is the case in Sakharov's induced gravity, in the standard derivation of the cosmological constant from vacuum effects, in the derivation of the Heisenberg-Euler effective free action for electromagnetism, mentioned above, etc. In contradistinction, in the MOND regime there is no experimental indication that inertia is instantaneous. In fact, as we saw, theoretical arguments lead us to expect that inertia is nonlocal.

It is clear from the undirected account given so far that I can offer no specific mechanism for inertia from vacuum. One vague notion that might be invoked is the Le Chatelier principle: Non-inertial motion is accompanied by a nontrivial manifestation of the vacuum. Inertia might then be an attempt of the vacuum to restore equilibrium and minimize the thermal effects of non-inertiality. One would like to find an action functional of the trajectory of the form (11) that embodies such an effect. The actual interaction with the vacuum will enter the mass factor $A_{m}$, while the "kinematic" factor that depends on the trajectory alone will come from the transformation of the vacuum to the 
non-inertial frame

\section{MOND AS A VACUUM EFFECT}

If the idea underlying MOND is basically correct, it will add a new dimension to the Machian concept of inertia. One will now have to explain the appearance of $a_{0}$ from more fundamental concepts, and the connection with cosmology. Beside the appearance of a cosmological parameter, MOND introduces two major changes: inertia becomes non-linear and, most probably, nonlocal.

Can we spot an inkling of MOND inertia in the Unruh effect? When the acceleration of a constant- $a$ observer becomes smaller than $a_{0}$, the typical frequency of its Unruh radiation becomes smaller than the expansion rate of the Universe, the Unruh wavelength becomes larger than the Hubble distance, etc. [5] [6]. We expect then some break in the response of the vacuum when we cross the $a_{0}$ barrier. It is interesting, as a preliminary indication, to understand the nature of the Unruh radiation seen by a non-inertial observer in a nontrivial universe. It is well known that even inertial observers in a nontrivial universe observe, generically, some sort of radiation arising from the distortion of the vacuum. The simplest and best studied case is that of a de Sitter universe where all inertial observers see a thermal spectrum with a temperature $T_{\Lambda}=(\Lambda / 3)^{1 / 3} / 2 \pi$ [26], where $\Lambda$ is the cosmological constant characterizing the de Sitter cosmology. It was shown recently [27] 28] that an observer on a constant-a (hyperbolic) trajectory, in a de Sitter universe also sees thermal radiation, but with a temperature

$$
T(a)=\frac{1}{2 \pi}\left(a^{2}+\Lambda / 3\right)^{1 / 2}
$$

de Sitter space-time can be embedded in a five-dimensional Minkowski space time. In the latter, the above observer moves in exact hyperbolic motion with the absolute value of the 5-acceleration

$$
a_{5}=\left(a^{2}+\Lambda / 3\right)^{1 / 2}
$$

The radiation that observer sees can thus be viewed as the Unruh radiation in the 5-D embedding space. It can be shown that relation (7) between the instantaneous, absolute value of the 4-acceleration in de Sitter space, and that in the embedding Minkowski space holds for an arbitrary trajectory.

Harking back to the Le Chatelier principle, if we envisage inertia as a force that drives a non-inertial body back to equilibrium as regards the vacuum radiation (here, drive $T$ back to $T_{\Lambda}$ ) then $T-T_{\Lambda}$ is a relevant quantity. We can write

$$
2 \pi\left(T-T_{\Lambda}\right) \equiv 2 \pi \Delta T=a \hat{\mu}\left(a / \hat{a}_{0}\right)
$$

with

$$
\hat{\mu}(x)=\left[1+(2 x)^{-2}\right]^{1 / 2}-(2 x)^{-1},
$$

and $\hat{a}_{0}=2(\Lambda / 3)^{1 / 2}$. The quantity $\Delta T$ behaves in just the manner required from MOND inertia [1], with $a_{0}=\hat{a}_{0}$ identified naturally with a cosmic acceleration parameter. [Note that $\hat{\mu}(x \ll 1) \approx x, \hat{\mu}(x \gg 1) \approx 1-(2 x)^{-1}$.] The significance of this suggestive finding is anything but obvious. First, it is not really clear why $\Delta T$ should be a measure of inertia ( similar quantities such as $T^{2}-T_{\Lambda}^{2}$ do not give the correct MOND behavior). Second, it is difficult to see how to generalize the argument to arbitrary motions. It would be particularly interesting to generalize it to nonrelativistic, circular motions, relevant to rotation-curve analysis of disk galaxies-the most clear-cut test of MOND to date. For circular orbits, all nonlocal inertia theories lead to an effective inertia force of the form $a \mu\left(a / a_{0}\right)-$ as in eq.(5) -where $\mu(x)$ need not take the same form as for the liner-motion case, but must still satisfy $\mu(x \ll 1) \approx x$, and $\mu(x \gg 1) \approx 1$.

Note here that the quantity $a \frac{\partial T}{\partial a}$, which measures, e.g., the temperature change under small dilatations of the orbit, also gives a MOND-like expression

$$
\begin{gathered}
a \frac{\partial T}{\partial a}=a \mu\left(a / a_{0}\right), \\
\mu(x)=x /\left(1+x^{2}\right)^{1 / 2},
\end{gathered}
$$


and $a_{0}=(\Lambda / 3)^{1 / 2}$, although the significance of this is, again, not clear.

In de Sitter space-time the expansion rate, the space-time curvature, and the cosmological constant are all the same. These parameters differ from each other in a general Friedmanian universe (the first two are not even constant) and so the above lesson learnt for the de Sitter case does not tell us which of the cosmological acceleration parameters is to be identified with $a_{0}$ in the real Universe.

The qualitative, phenomenological tenet of MOND whereby inertia vanishes in the limit $a_{0} \rightarrow \infty$ can be understood in the present picture as follows. The limit corresponds to $\Lambda \rightarrow \infty$, or $H_{0} \rightarrow \infty$, etc.; so, the Gibbons-Hawking-like radiation due to cosmology swamps the thermal effects due to non-inertial motion such that the difference between inertial and non-inertial observers is erased in this limit.

As already noted above, the cosmological connection need not enter as a causative effect of cosmology on local dynamics-mediated by the vacuum). It may be that vacuum effects enter both cosmology-e.g. through a cosmological constant-and local dynamics through $a_{0}$, thus establishing the "cosmic coincidence" seen in MOND.

\section{ACKNOWLEDGMENTS}

I thank Jacob Bekenstein for many helpful discussions

[1] M. Milgrom, Astrophys. J. 270365 (1983)

[2] R.H. Sanders, Astrophys. J. 473117 (1996)

[3] S.S. McGaugh and W.J.G. de Blok, Astrophys. J. 49966 (1998)

[4] R.H. Sanders and M.A.W Verheijen, Astrophys. J. 50397 (1998)

[5] M. Milgrom, Comments on Astrophysics 13(4), 215 (1989)

[6] M. Milgrom, Ann. Phys. 229, 384 (1994)

[7] S. Perlmuter et al., preprint astro-ph/9812133 (1998)

[8] G. Efstathiou, S. L. Bridle A. N. Lasenby, M. P. Hobson, and R. S. Ellis, preprint astro-ph/9812226 (1998)

[9] J. Bekenstein and M. Milgrom, Astrophys. J. 2867 (1984)

[10] J.B. Barbour and H. Pfister (eds.), Mach's Principle, Birkhauser (Boston) (1995)

[11] Ya. B. Zeldovich, Pis'ma Zh. Eksp. Teoret. Fiz. 6, 922 (English translation in JETP Lett. 6, 345 1967)

[12] S.L. Adler, Rev. Mod. Phys. 54, 729 (1982)

[13] C. Itzykson and J.B. Zuber, Quantum Field Theory, McGraw-Hill (1980)

[14] A.D. Sakharov, Sov. Phys. Doklady, 12, 1040 (1968)

[15] A. Rueda and B. Haisch, Physics Letters A 240, 115 (1998)

[16] M.T. Jaekel and S. Reynaud, J. de Physique 3, 1093 (1993)

[17] B. Haisch, A. Rueda, and H.E. Puthoff, Phys. Rev. A 49, 678 (1994)

[18] W.G. Unruh, Phys. Rev. D 14, 870 (1975)

[19] N.D. Birell and P.C.W. Davies, Quantum Fields in Curved Space, Cambridge University Press (Cambridge), 1982

[20] U.H. Gerlach, Phys. Rev. D 27, 2310 (1983)

[21] J. Audretsch, R. Müller, and M. Holzmann Class. Quantum Grav. 122927 (1995)

[22] J.S. Bell and J.M. Leinaas, Nuc. Phys. B284, 488 (1987)

[23] O. Levin, Y. Peleg, and A. Peres, J. Phys. A: Math Gen. 26, 3001 (1993)

[24] W.G. Unruh, preprint hep-th/9804158 (1998)

[25] J.M. Leinaas, preprint hep-th/9804179 (1998)

[26] G.W. Gibbons and S.W. Hawking, Phys. Rev. D 15, 2738 (1977)

[27] H. Narnhofer, I. Peter, and W. Thirring, Int. J. Mod. Phys. B 101507 (1996)

[28] S. Deser and O. Levin, Class. Quant. Grav. 14, L163 (1997) 